\section{The Mathematical Work of the 2002 Fields Medalists}

\section{The Work of Laurent Lafforgue}

\section{by Michael Rapoport}

Laurent Lafforgue was awarded the Fields Medal for his proof of the Langlands correspondence for the general linear groups $G L_{r}$ over function fields of positive characteristic. His approach to this problem follows the basic strategy introduced twenty-five years ago by V. Drinfeld in his proof for $G L_{2}$. Already Drinfeld's proof is extremely difficult. Lafforgue's proof is a real tour de force, taking up as it does several hundred pages of highly condensed reasoning. By his achievement Lafforgue has proved himself a mathematician of remarkable strength and perseverance.

In this brief report I will sketch the background of Lafforgue's result, state his theorems, and then mention some ingredients of his proof. The final passages are devoted to the human factor.

\section{The Background}

The background of Lafforgue's theorem is the web of conjectures known as the Langlands philosophy, which is a far-reaching generalization of class field theory. Let $F$ be a global field, i.e., either a finite extension of $\mathbf{Q}$ (the number field case) or a finite extension of $\mathbf{F}_{p}(t)$ where $\mathbf{F}_{p}$ is the finite field with $p$ elements (the function field case). Let $\mathbf{A}$ be the adèle ring of $F$.

Global class field theory may be formulated as giving a bijection between the sets of characters of finite order of the Galois group $\operatorname{Gal}(\bar{F} / F)$ on the one hand and of the idèle class group $\mathbf{A}^{\times} / F^{\times}$on the other hand. This is the reciprocity law of T. Takagi and E. Artin established in the 1920s as a farreaching generalization of the quadratic reciprocity law going back to L. Euler. At the end of the 1960s, R. Langlands proposed a nonabelian generalization of this reciprocity law. It conjecturally relates the irreducible representations of rank $r$ of $\operatorname{Gal}(\bar{F} / F)$ (or, more generally, of the hypothetical motivic Galois

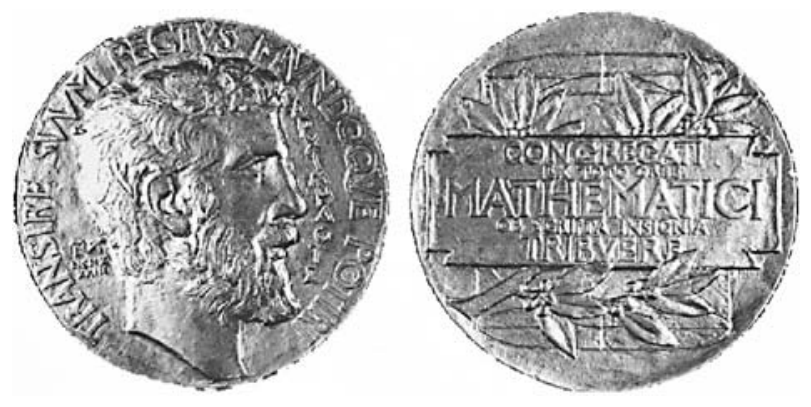

group of $F$ ) with cuspidal automorphic representations of $G L_{r}(\mathbf{A})$. In fact, this conjecture is part of an even grander panorama of Langlands (the functoriality principle), in which homomorphisms between $L$ groups of reductive groups over $F$ induce relations between the automorphic representations on the corresponding groups. These hypothetical reciprocity laws would imply famous conjectures, such as the Artin conjecture on the holomorphy of $L$-functions of irreducible Galois representations and the RamanujanPetersson conjecture on the Hecke eigenvalues of cusp forms for $G L_{r}$.

In the number field case, deep results along these lines have been obtained for groups of small rank, such as $G L_{2}$, by Langlands himself and by many others. And such results have already had spectacular applications, such as in the proof of Fermat's last theorem. However, the proof of the Langlands correspondence in any generality in the number field case seems out of reach at the present time. Lafforgue's result, which concerns the function field case, is the first general nonabelian reciprocity law.

\section{Lafforgue's Theorem}

From now on let $F$ denote a function field of characteristic $p$. We also fix an auxiliary prime number $\ell \neq p$. For a positive integer $r$ let $\mathcal{G}_{r}$ be the set of equivalence classes of irreducible $\ell$-adic representations of dimension $r$ of $\operatorname{Gal}(\bar{F} / F)$. For each $\sigma \in \mathcal{G}_{r}$, A. Grothendieck defined its $L$-function $L(\sigma, s)$, which is a rational function in $p^{-s}$ satisfying a functional equation of the form $L(\sigma, s)=\varepsilon(\sigma, s) \cdot L\left(\sigma^{\vee},-s\right)$, where $\varepsilon(\sigma, s)$ is a monomial in $p^{-s}$ and $\sigma^{\vee}$ denotes the contragredient representation. The $L$-function is an Euler product, $L(\sigma, s)=\prod_{x} L_{x}(\sigma, s)$, over all places $x$ of $F$, and for a place $x$ of degree $\operatorname{deg}(x)$, where $\sigma$ is unramified, we have

$$
L_{x}(\sigma, s)=\prod_{i=1}^{r} \frac{1}{1-z_{i} p^{-s \operatorname{deg}(x)}} .
$$

Here $z_{1}, \ldots, z_{r}$ are the Frobenius eigenvalues of $\sigma$ at $x$. 


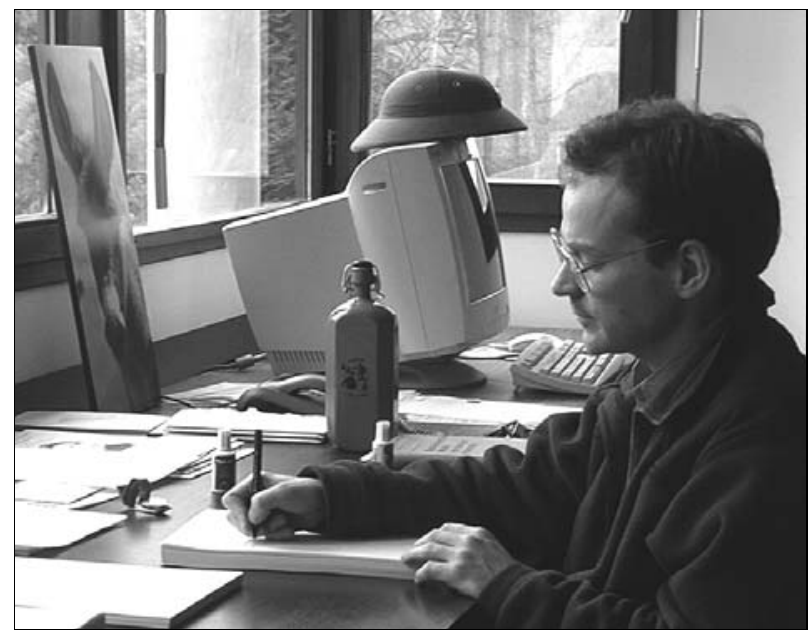

Let $\mathcal{A}_{r}$ be the set of equivalence classes of cuspidal representations of $G L_{r}(\mathbf{A})$. For each $\pi \in \mathcal{A}_{r}$, R. Godement and H. Jacquet defined its $L$-function $L(\pi, s)$ with properties similar to those of the above $L$-functions. The Euler factor at a place $x$ where $\pi$ is unramified is given as

$$
L_{x}(\pi, s)=\prod_{i=1}^{r} \frac{1}{1-z_{i} p^{-s \operatorname{deg}(x)}}
$$

where $z_{1}, \ldots, z_{r}$ are the Hecke eigenvalues of $\pi$ at $x$. The main result of Lafforgue consists of the following theorems.

Theorem 1 (The LAnglands CONJECture). There is a bijection $\pi \mapsto \sigma(\pi)$ between $\mathcal{A}_{r}$ and $\mathcal{G}_{r}$, characterized by the fact that $L_{x}(\pi, s)=L_{x}(\sigma(\pi), s)$ for every place $x$ of $F$.

Theorem 2 (The Ramanujan-Petersson CONJECTURE). Let $\pi \in \mathcal{A}_{r}$ with central character of finite order. Then for every place $x$ of $F$ where $\pi$ is unramified, the Hecke eigenvalues $z_{1}, \ldots, z_{r} \in \mathbf{C}$ are all of absolute value 1 .

Theorem 3 (The Deligne Conjecture). Let $\sigma \in \mathcal{G}_{r}$ with determinant character of finite order. Then $\sigma$ is pure of weight 0, i.e., for any place $x$ of $F$ where $\sigma$ is unramified, the images of the Frobenius eigenvalues $z_{1}, \ldots, z_{n}$ under any embedding of $\overline{\mathbf{Q}}_{\ell}$ into $\mathbf{C}$ are of absolute value 1 .

Here Theorems 2 and 3 are consequences of Theorem 1 through P. Deligne's purity theorem and the estimate on Hecke eigenvalues of Jacquet and J. Shalika. Theorem 1 itself is proved by induction on $r$ (Deligne recursion principle). After what was known before (in addition to the functional equations, essentially the converse theorems of A. Weil and I. PiatetskiiShapiro and the product formula for $\varepsilon$-factors of G. Laumon), it all boiled down to proving the existence of the map $\pi \mapsto \sigma(\pi)$ with the required properties. This is exactly what Lafforgue did.
Before spending a few words on his proof, let us consider the question, What is it good for? The answer is that neither of the sets $\mathcal{G}_{r}$ and $\mathcal{A}_{r}$ is simpler than the other in every aspect but that Theorem 1 can be used to transfer available information in either direction. Theorem 3 is an instance where information available on $\mathcal{A}_{r}$ implies results on $\mathcal{G}_{r}$. In the other direction, Theorem 1 permits one to use constructions available on $\mathcal{G}_{r}$ to prove various instances of Langlands functoriality for $\mathcal{A}_{r}$, such as the existence of tensor products, of base change, and of automorphic induction.

\section{About the Proof}

The strategy of constructing the map $\pi \mapsto \sigma(\pi)$ is due to Drinfeld and is inspired by the work of Y. Ihara, Langlands, and others in the theory of Shimura varieties. It consists in analyzing the $\ell$-adic cohomology of the algebraic stack $\operatorname{Sht}_{r, \varnothing}$ over $\operatorname{Spec} F \times \operatorname{Spec} F$ parametrizing shtukas of rank $r$ or the algebraic stack Sht $_{r}=\lim _{\leftarrow}$ Sht $_{r, N}$ parametrizing shtukas of rank $r$ equipped with a compatible system of level structures for all levels $N$. The latter cohomology module is equipped with an action of $G L_{r}(\mathbf{A}) \times \operatorname{Gal}(\bar{F} / F) \times$ $\operatorname{Gal}(\bar{F} / F)$, and the aim is to isolate inside it a subquotient of the form

$$
\bigoplus_{\pi \in \mathcal{A}_{r}} \pi \otimes \sigma(\pi) \otimes \sigma(\pi)^{\vee}
$$

by comparing the Grothendieck-Lefschetz fixedpoint formula and the Arthur-Selberg trace formula. The essential difficulty is that, in contrast to the case of Shimura varieties, the moduli stack Sht $r$ is not of finite type, not even at any finite level $N$. To understand why, recall that a shtuka of rank $r$ is a vector bundle of rank $r$ on $X$ with additional structure (essentially a meromorphic descent datum under Frobenius). Here $X$ is the smooth irreducible projective curve over $\mathbf{F}_{p}$ with function field $F$. And, just as the moduli stack of vector bundles of rank $r$ on $X$ is not of finite type, neither are the stacks Sht $_{r, \varnothing}$ and Sht $_{r, N}$.

To deal with this difficulty, Lafforgue introduces the open substacks Sht $\underset{r, \varnothing}{\leq P}$ and $\mathrm{Sht}_{r, N} \leq P$ of shtukas where the Harder-Narasimhan polygon is bounded by $P$. These substacks are of finite type, and their union is the whole space. The trouble is that they are not stable under the Hecke correspondences. Therefore Lafforgue constructs in the case without level structure a smooth compactification $\overline{\mathrm{Sht}_{r, \varnothing}^{\leq P}}$ of $\mathrm{Sht}_{r, \varnothing}^{\leq P}$ with a normal crossing divisor at infinity and extends the Hecke correspondences to it bysimple normalization. He then applies the Grothendieck-Lefschetz fixed-point formula to these correspondences. However, only a part of this formula can be determined 
explicitly, and therefore this seems a pointless exercise. Lafforgue circumvents this problem by isolating the $r$-essential part of the cohomology of Sht ${ }_{r, \varnothing}^{\leq P}$ and by showing that the remainder, both the difference between the cohomology of Sht ${ }_{r, \varnothing}^{\leq P}$ and of Sht $r, \varnothing$ and the cohomology of the boundary of $\mathrm{Sht}_{r, \varnothing}^{\leq P}$, is $r$ negligible. Here the work of R. Pink on Deligne's conjecture on the Grothendieck-Lefschetz formula enters in a decisive way. In the case where a level structure is imposed, Lafforgue manages to push through his method by constructing a partial compactification of $\mathrm{Sht}_{r, N}^{\leq P}$ which is smooth with a normal crossing divisor at infinity and which is stable under the Hecke correspondences and by supplementing Pink's theorem by K. Fujiwara's theorem.

\section{The Months of Suspense}

Lafforgue's first attempt at a proof of Theorem 1 used a compactification of $\mathrm{Sht}_{r, N}^{\leq P}$. His construction was based on the compactifications of the quotient spaces $X_{r, n}=\left(P G L_{r}\right)^{n+1} / P G L_{r}$ that he had defined in earlier work, generalizing the case $n=1$ due to C. De Concini and C. Procesi. In June 2000, while lecturing on his proof, Lafforgue discovered that, contrary to what he had claimed, these compactifications of $X_{r, n}$, and hence also the corresponding compactifications of Sht ${ }_{r, N}^{\leq P}$, are not smooth in general. He was not even able to resolve their singularities. During two months of suspense in the summer of 2000, Lafforgue managed to fill the gap by finding the above-mentioned partial compactifications of $\mathrm{Sht}_{r, N}^{\leq P}$ and was able to finesse the proof of Theorem 1 from them. Thus in the end, the modified argument is simpler than the original attempt.

Even though Lafforgue's compactifications of $X_{r, n}$ are not used in the final proof, they are fascinating objects in themselves, with close relations to such diverse geometric objects as configuration spaces of matroids, thin Schubert cells, stable degeneration of $n$ pointed projective lines, and local models of Shimura varieties. It turns out that these compactifications are smooth for $n=1$ (respectively toroidal for $n=2$ ) and arbitrary $r$ (De Concini and Procesi, respectively Lafforgue) and for $r=2$ and arbitrary $n$ (G. Faltings), but can have arbitrarily bad singularities in general (N. Mnëv). These compactifications constitute a new field of investigation, taken up by Lafforgue in a 265-page preprint (http://www . ihes.fr/ PREPRINTS/M02/Resu/resu-M02-31.html).

\section{Biographical Data}

Laurent Lafforgue was born in 1966. He was a student at the École Normale Supérieure (1986-1990) before entering the Centre National des Recherches Scientifiques in 1990. His academic teacher is Gérard
Laumon, with whom he obtained his thèse at the Université de Paris-Sud in 1994. It is in the famous Bâtiment de Mathématique ("le 425") on the Orsay campus that Lafforgue worked out his proof. Since 2000 he has been a professor at the Institut des Hautes Études Scientifiques.

\section{Further Information}

For excellent overviews of Lafforgue's proof, see Laumon's Bourbaki seminar No. 873, March 2000, which also contains an annotated bibliography, and Lafforgue's notes of a course at the Tata Institute (http://www.ihes.fr/PREPRINTS/M02/Resu/ resu-M02-45.html).

\section{The Work of Vladimir Voevodsky}

\section{by Eric M. Friedlander and Andrei Suslin}

In 1982 Alexander Beilinson stated conjectures which crystallized a vision of the relationship between algebraic $K$-theory and "integral motivic cohomology theory" of algebraic varieties over a field $F$ and between mod- $\ell$ algebraic $K$-theory and mod- $\ell$ étale cohomology, where $\ell$ is a prime invertible in $F[\mathrm{Be}]$. These conjectures, more detailed and specific than the earlier "Quillen-Lichtenbaum Conjecture", provided a challenging program that explains a great deal about Quillen's algebraic $K$-theory [Q]. Although one of Beilinson's conjectures remains unsolved (and may well prove to be false in general), Vladimir Voevodsky has made great strides in completing Beilinson's program.

Voevodsky's achievements are remarkable. First, he has developed a general homotopy theory for algebraic varieties. Second, as part of this general theory, he has formulated what appears to be the "correct" motivic cohomology theory and verified many of its remarkable properties. Third, as an application of this general approach, he has proved a longstanding conjecture of John Milnor relating the Milnor $K$-theory of a field to its étale cohomology (and to quadratic forms over the field).

We provide below a brief sketch of Voevodsky's contributions, proceeding from the specific Milnor Conjectures to the more general theory.

\section{Milnor Conjecture}

Given a field $F$, we define (following Milnor) the graded ring $K_{*}^{M}(F)$ to be the tensor algebra over the 
integers $\mathbb{Z}$ of the group $F^{*}$ of nonzero elements in $F$, modulo the ideal generated by the Steinberg relations in $F^{*} \otimes F^{*}$ (so that $\{a, 1-a\}$, the image of $a \otimes(1-a)$, is set equal to $0 \in K_{2}^{M}(F)$ for all $\left.a \neq 1 \in F^{*}\right)$. Thus, $K_{0}^{M}(F)=K_{0}(F)=\mathbb{Z}, K_{1}^{M}(F)=K_{1}(F)=F^{*}$, and (thanks to a theorem of Matsumoto) $K_{2}^{M}(F)=$ $K_{2}(F)=F^{*} \otimes F^{*} /\left(a \otimes(1-a) ; a \neq 1 \in F^{*}\right)$. By definition, the $n$-th Milnor $K$-group $K_{n}^{M}(F)$ is generated by products of elements in degree 1 (so-called symbols $\left.\left\{a_{1}, \ldots, a_{n}\right\}\right)$, thereby being much simpler than Quillen's $K$-group $K_{n}(F)$.

The following theorem, conjectured by Milnor [Mi], has been proved by Voevodsky.

THEOREM 1. [V4] Let $F$ be a field of characteristic different from 2. Then the natural map

$$
K_{*}^{M}(F) \otimes \mathbb{Z} / 2 \mathbb{Z} \rightarrow H^{*}(F, \mathbb{Z} / 2 \mathbb{Z})
$$

is an isomorphism of graded rings. In particular, for every $n>0$, the Galois cohomology group $H^{n}(F, \mathbb{Z} / 2 \mathbb{Z})$ is generated by $n$-fold cup products of cohomology classes of degree 1.

One can marvel at this result from many points of view. For example, it says there is something very special about graded rings that arise as Galois cohomology of fields.

Voevodsky, in collaboration with D. Orlov and A. Vishik, has also proved the following closely related theorem conjectured by Milnor [Mi]. The Grothendieck-Witt ring $G W(F)$ has an underlying abelian group given by isomorphism classes of finite-dimensional $F$-vector spaces equipped with a quadratic form and ring structure given by tensor product. The Witt ring $W(F)$ is obtained by dividing $G W(F)$ by the ideal generated by the 2-dimensional $F$-vector space with the hyperbolic quadratic form. The Witt ring $W(F)$ admits an augmentation map $W(F) \rightarrow \mathbb{Z} / 2 \mathbb{Z}$ induced by the rank $\operatorname{map} G W(F) \rightarrow \mathbb{Z}$. Milnor investigated the successive quotients $I^{n} / I^{n+1}$, where $I \subset W(F)$ denotes the kernel of the augmentation map, and the resulting associated graded ring $g r_{*}(W(F))=\bigoplus_{n \geq 0} I^{n} / I^{n+1}$.

TheOREM 2. [OVV] Let $F$ be a field of characteristic different from 2. Then the natural homomorphism of graded rings $K_{*}(F) \otimes \mathbb{Z} / 2 \mathbb{Z} \rightarrow g r_{*}(W(F))$ is an isomorphism.

Theorems 1 and 2 show that the three graded $\operatorname{rings} K_{*}^{M}(F) \otimes \mathbb{Z} / 2 \mathbb{Z}, g r_{*}(W(F))$, and $H^{*}(F, \mathbb{Z} / 2 \mathbb{Z})$, which arise in very different manners, are canonically isomorphic for any field of characteristic different from 2.

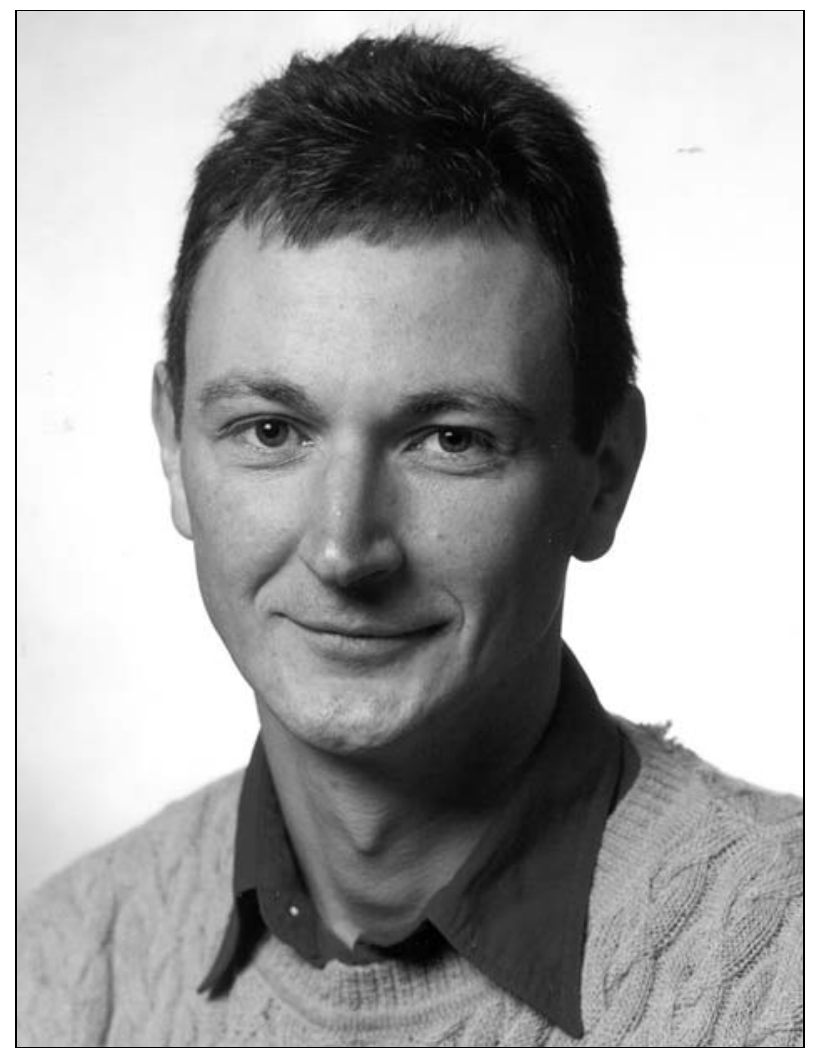

\section{Motivic Cohomology}

In algebraic topology, singular cohomology with integral coefficients has many good properties and satisfies a tight relationship with topological $K$-theory. One has known for many years that one cannot define "algebraically" the integral (or even rational) singular cohomology of complex algebraic varieties. On the other hand, étale cohomology with mod- $\ell$ coefficients, as developed by Alexander Grothendieck and Michael Artin, when applied to varieties over a field $F$, succeeds admirably in providing a substitute for singular cohomology mod- $\ell$ whenever $\ell$ does not equal the characteristic of $F$.

Beilinson conjectured the existence of motivic complexes $\mathbb{Z}(q)$ leading to bigraded motivic cohomology groups $H^{p}(X, \mathbb{Z}(q))$ which should relate to the algebraic $K$-theory of a smooth variety $X$ over a field $F$. (This relationship has recently seen various proofs, beginning with work of Bloch-Lichtenbaum, then Friedlander-Suslin, Levine, and finally GraysonSuslin.) Moreover, he conjectured that these complexes, when reduced modulo $\ell$ for $\ell$ invertible in $F$, should be related in a completely precise way to étale cohomology.

In [Bl] Spencer Bloch produced an ingenious construction yielding "higher Chow groups", which are excellent candidates for the cohomology of Beilinson's conjectured motivic complexes. Somewhat later, 
Suslin introduced algebraic singular complexes which led Suslin and Voevodsky to an alternate algebraic model of singular cohomology with mod- $\ell$ coefficients [SV1]. A major achievement of Voevodsky has been to formulate a natural category (of abelian presheaves with transfers on the big Nisnevich site of all smooth varieties $X$ over $F$ ) which in conjunction with the algebraic singular complex construction leads to a good formulation of motivic complexes $\mathbb{Z}(n)$ developed by Suslin and Voevodsky [SV2]. Recently Voevodsky has proved that the resulting cohomology groups are isomorphic to Bloch's higher Chow groups [V2].

Voevodsky's formulation of motivic cohomology is crucial, for it has led Voevodsky to prove various important properties essential for his proof of the Milnor Conjectures. We mention perhaps the most important general theorem concerning motivic cohomology, a theorem for which Voevodsky has recently found a most elegant proof.

Theorem 3. [FV] [V5] Let $X$ be a smooth variety over a field $F$, and let $Y \subset X$ be a smooth closed subvariety everywhere of codimension $d$. Then there is a canonical Gysin isomorphism

$$
H_{Y}^{p}(X, \mathbb{Z}(n))=H^{p-2 d}(Y, \mathbb{Z}(n-d))
$$

Operations in Motivic Cohomology and the Proof of the Milnor Conjecture

Steenrod operations in mod- $\ell$ motivic cohomology are at the heart of Voevodsky's proof of the Milnor Conjecture. The construction of these operations given in [V3] turned out to be much more subtle than the topological counterpart, and their properties in the special case $\ell=2$ differ somewhat from the corresponding properties of Steenrod operations in topological singular cohomology.

Following the approach for $n=2$ introduced by A. Merkurjev and Suslin, Voevodsky investigated what happens when one splits a symbol $\left\{a_{1}, \ldots, a_{n}\right\} \in$ $K_{n}^{M} \otimes \mathbb{Z} / \ell$. For any such symbol, there exists a universal splitting variety $X_{a}$. Earlier work of Suslin and Voevodsky [SV2], together with several ingenious arguments introduced by Voevodsky, reduces the proof of the Milnor Conjecture (and the more general Bloch-Kato Conjecture) to a specific vanishing calculation in motivic cohomology associated to $X_{a}: H^{n+1}\left(\check{C}\left(X_{a}\right), \mathbb{Z}_{(\ell)}(n)\right)=0$, where $\check{C}\left(X_{a}\right)$ is a simplicial scheme with $C_{n}\left(X_{a}\right)=X_{a}^{n+1}$ and all face (respectively, degeneracy) maps given by projections (respectively, diagonal embeddings).

Associated to Voevodsky's Steenrod operations are the corresponding Milnor operations

$Q_{i}: \widetilde{H}^{p}(-, \mathbb{Z} / \ell(q)) \rightarrow \widetilde{H}^{p+2 \ell^{i}-1}\left(-, \mathbb{Z} / \ell\left(q+\ell^{i}-1\right)\right)$.
As in algebraic topology, these operations satisfy the property $Q_{i}^{2}=0$, so motivic cohomology provided with the operator $Q_{i}$ forms a complex, whose homology is known as Margolis homology. As a next step, Voevodsky proved the following remarkable theorem concerning the vanishing of Margolis homology.

Theorem 4. [V3] Let $X$ be a smooth projective variety over a field $k$ of characteristic different from $\ell$. Assume that there exists a morphism $Y \rightarrow X$ from a smooth projective variety $Y$ of dimension $\ell^{m}-1$ to $X$, and further assume that the characteristic number $\operatorname{deg}\left(s_{\ell^{m}-1}(Y)\right)$ is not congruent to 0 modulo $\ell^{2}$. Then all of the Margolis homology groups of the simplicial sheaf $\widetilde{C}(X)$ (the unreduced suspension of $\check{C}(X)$ ) corresponding to the operation $Q_{m}$ are 0 .

For $\ell=2$, one can represent the splitting variety $X_{a}$ by the quadric $\mathcal{Q}_{q}$ defined by the Pfister neighbor $q=\left\langle\left\langle a_{1}, \ldots, a_{n-1}\right\rangle\right\rangle \perp\left\langle-a_{n}\right\rangle$ of $\left\langle\left\langle a_{1}, \ldots, a_{n}\right\rangle\right\rangle$, which is a smooth projective variety of dimension $2^{n-1}-1$ and satisfies the condition that $\operatorname{deg}\left(s_{2^{n}-1}\left(X_{a}\right)\right)$ is not divisible by 4 . We now apply Theorem 4 with $X=X_{a}$ and $Y=\mathcal{Q}_{\left\langle\left\langle a_{1}, \ldots, a_{m-1}\right\rangle\right\rangle \perp\left\langle-a_{n}\right\rangle}$ for $1 \leq m \leq$ $n$ to conclude the vanishing of the Margolis homology of $\widetilde{C}\left(X_{a}\right)$ with respect to each of the operations $Q_{1}, \ldots, Q_{n}$.

Using dimension considerations, Voevodsky observes that the operation $Q_{n-2} \circ \cdots \circ Q_{1}$ and its integral counterpart

$$
\begin{aligned}
\widetilde{Q}_{n-2} \circ \cdots \circ \widetilde{Q}_{1}: \widetilde{H}^{n+2}\left(\widetilde{C}(X), \mathbb{Z}_{(2)}(n)\right) \\
\left.\rightarrow \widetilde{H}^{2^{n}}\left(\widetilde{C}(X), \mathbb{Z}_{(} 2\right)\left(2^{n-1}\right)\right)
\end{aligned}
$$

are monomorphisms. This reduces the proof of the vanishing of $H^{n+1}\left(\check{C}(X) \mathbb{Z}_{(2)}(n)\right)$, to the vanishing of the group $H^{2 n-1}\left(\check{C}(X) \mathbb{Z}_{(2)}\left(2^{n-1}\right)\right)$. Fortunately the latter group is much easier to understand: Using [R1], Voevodsky shows that this group is closely related to the group of 0 -dimensional $K_{1}$-cycles studied closely by M. Rost in [R2]. To finish the proof, Voevodsky then applies the main theorem of [R2].

\section{The Homotopy Category of Schemes}

Beginning with his Harvard Ph.D. thesis, Voevodsky has had the goal of creating a homotopy theory for algebraic varieties amenable to calculations as in algebraic topology. Much of this homotopy theory, both stable and unstable, has been developed in collaboration with Fabien Morel (see [MV]). In this abstract context one can realize motivic cohomology and algebraic $K$-theory as representable functors. One can view motivic Steenrod operations as a special case of operations on "generalized stable cohomology theories". When Voevodsky localizes to obtain a homotopy category of schemes, he is forcing "homotopy 
invariance", the property that a homotopy type (e.g., represented by a scheme) is viewed as equivalent to the product of itself and the affine line. A key insight of Voevodsky is that there are two types of "circles" in algebraic geometry determining two types of suspension. One type of circle arises when one considers an affine nodal curve (tracing out a curve which crosses itself) and the other when one considers the punctured affine line (which is simply real 2-space minus the origin in the special case the ground field is the complex numbers).

Although Voevodsky has given us a relatively streamlined proof of the Milnor Conjecture which does not rely on this homotopy category, his original conception of the proof relied heavily on such a homotopytheoretic point of view. Moreover, the expected proof of the odd-prime analogue of the Milnor Conjecture (the so-called "Bloch-Kato Conjecture") fully utilizes this appealing formalism.

We conclude by observing that not only has Voevodsky's work much influenced how algebraic geometers are approaching certain classical questions but also algebraic topologists have begun to produce considerable foundational material in this new homotopy theory.

\section{References}

[Be] A. Beilinson, letter to C. Soulé, 1982.

[Bl] S. Bloch, Algebraic cycles and higher $K$-theory, Adv. in Math. 61 (1986), 267-304.

[FV] E. Friedlander and V. Voevodsky, Bivariant cycle cohomology, Cycles, transfers, and Motivic Homology theories, Ann. of Math. Stud., vol. 143, Princeton Univ. Press, Princeton, NJ, 2000, 138-87.

[Mi] J. Milnor, Algebraic -theory and quadratic forms, Invent. Math. 9 (1970), 318-44.

[MV] F. Morel and V. Voevodsky, $\mathbb{A}^{1}$-homotopy theory of schemes, Inst. Hautes Études Sci. Publ. Math. No. 90 (1999), 45-143 (2001).

[OVV] D. Orlov, A. Vishik, and V. Voevodsky, An exact sequence for Milnor's $K$-theory with applications to quadratic forms, 2000 eprint, http://www.math.uiuc. edu/K-theory/0454/, also arXiv: math.AG/0101023.

[Q] D. Quillen, Higher Algebraic $K$-Theory: I, Lecture Notes in Math., vol. 341, Springer, Berlin, 1973, pp. 85-147.

[R1] M. Rost, The motive of a Pfister form, 1998 eprint, http://www.math.ohio-state. edu/ rost/motive.html.
[R2] - Some new results on the Chow groups of quadrics, 1990 eprint, http://www.math.ohio-state.edu/ rost/ chowqudr.html.

[SV1] A. Suslin and V. Voevodsky, Singular homology of abstract algebraic varieties, Invent. Math. 123 (1996), 6194.

[SV2] —, Bloch-Kato conjecture and motivic cohomology with finite coefficients, The Arithmetic and Geometry of Algebraic Cycles, NATO Sci. Ser. C Math. Phys. Sci., vol. 548, Kluwer, Dordrecht, 2000, pp. 117-89.

[V1] V. Voevodsky, Cohomological theory of presheaves with transfers, Cycles, Transfers, and Motivic Homology Theories, Ann. of Math. Stud., vol. 143, Princeton Univ. Press, Princeton, NJ, 2000, pp. 87-137.

[V2] -, Motivic cohomology groups are isomorphic to higher Chow groups in any characteristic, Int. Math. Res. Not. no. 7 (2002), 351-5.

[V3] - , Reduced power operations in motivic cohomology, 2001 eprint, http://www.math.uiuc.edu/K-theory/ 0487/, also arXiv:math.AG/0107109.

[V4] -, On 2-torsion in motivic cohomology, 2001 eprint, http://www.math. uiuc.edu/K-theory/0502/, also arXiv:math.AG/0107110.

[V5] -, Cancellation theorem, 2002 eprint, http://www. math.uiuc.edu/K-theory/0541/, also arXiv:math.AG/ 0202012 .

\section{Authors addresses}

Prof. Dr. Michael Rapoport

Mathematisches Institut

Universität zu Köln

Weyertal 86-90

$50931 \mathrm{Köln}$

rapoport@math.uni-koeln.de

Prof. Dr. Eric M. Friedlander

Department of Mathematics

Northwestern University

Evanston, IL 60208-2730, USA

eric@math.nwu.edu

Prof. Dr. Andrei Suslin

Department of Mathematics

Northwestern University

2033 Sheridan Road

Evanston, IL 60208-2730, USA

suslin@math.northwestern.edu

(Zuerst in: Notices of the AMS, vol. 50 (2), 2003, 212-217. Bebilderung: Mitteilungen) 